\title{
AN INTEGER ASSOCIATED WITH LATIN SQUARES
}

\author{
D. A. NORTON AND SHERMAN K. STEIN
}

1. Introduction. This paper introduces a new number associated with a wide class of Latin squares. By the methods of combinatorial topology, suggested in [1], it is shown in Theorem II that the parity of this number is determined by the order of the square.

Let a diagonalized Latin square be a Latin square in which the main diagonal (the diagonal extending from the upper left corner to the lower right corner) is the $n$ elements in their natural order. Consider the set of $n^{2}$ triples $(i, j, a)$ associated with such a square $A$ where $i$ is the row index, $j$ the column index, and $a$ the entry in the $(i, j)$ th position in the square.

Lemma. A triplet is determined by any two of its coordinates.

To prove this lemma, define the orthogonal projection $\pi_{i}$ as the operator which maps each 3-tuple on a 2-tuple by eliminating the $i$ th coordinate. Then if $t$ is any triplet, $\pi_{i} t, i=1,2,3$, is an ordered pair of integers. Since $A$ is a Latin square, the set $\left\{\pi_{i} t\right\}$, as $t$ ranges over all the triplets, is the complete set of all possible $n^{2}$ ordered number pairs of $n$ distinct integers. If $t$ and $t^{\prime}$ are distinct triplets, $\pi_{i} t \neq \pi_{i} t^{\prime}$ since there are only $n^{2}$ pairs in the set $\left\{\pi_{i} t\right\}$ and they must all be distinct.

Because of the diagonal property of $A$, each of the triplets $(k, k, k)$, $k=1,2, \cdots, n$, must appear. If these are deleted first from the set of triplets, the set of images $\pi_{i} t$ will be the possible $n(n-1)$ ordered pairs of distinct numbers.

The mapping $\pi_{i}$ is 1-1 so the mapping $\pi_{i}^{-1}$ is single-valued.

THEOREM I. There is a natural correspondence between diagonalized Latin squares and sets of $n(n-1)$ triplets $t$ satisfying:

(i) the projection $\pi_{i}, i=1,2,3$ is one to one;

(ii) there are no fixed triplets under the mapping $\pi_{i}^{-1} T \pi_{i}=P_{i}$ where $T$ is transposition.

To complete the proof of the theorem it is only necessary to note that to a set of $n(n-1)$ triplets, with the properties (i) and (ii), the adjunction of triplets $(k, k, k), k=1, \cdots, n$, yields a set of $n^{2}$ triplets from which a diagonalized Latin square may be constructed.

Received by the editors May 4, 1955. 
Since the mappings $P_{i}$ of Theorem I are self-inverse, they induce a symmetric relation $R$ between triplets. The subset of triplets, all of which contain a fixed integer $m$, may be divided into equivalence classes (depending on the integer $m$ ) in which two triplets $s$ and $t$ are equivalent if there exists a chain of triplets $t_{1}, \cdots, t_{k}$ such that $s=t_{1}, t_{1} R t_{2}, \cdots, t_{k-1} R t_{k}, t_{k}=t$. Each such equivalence class will be called a cycle. Define the length of a cycle to be the number of distinct triplets contained in it.

It may be observed that the length of a cycle is divisible by 3 . To prove this, a cycle may be divided into 3 sets $M_{i}, i=1,2,3$, where $m$ appears as the $i$ th coordinate in each triple of $M_{i}$. From the definition of $P_{i}$ it is true that $P_{i} M_{j}=M_{k}, i, j, k$ all different. Since $P_{i}$ is one to one, each set $M_{i}$ contains the same number of elements. The observation follows since the sets $M_{i}$ do not intersect.

It is also of interest to note that two cycles on different integers may intersect without being identical but two cycles on the same integer can intersect only if they are identical. However there may be several distinct cycles on one integer. Let $\Phi(m)$ be the number of cycles on the integer $m$ and let $Z=\sum_{m} \Phi(m)$. Clearly $n \leqq Z \leqq n(n-1)$. $\$ 2$ contains a proof of:

Theorem II. $Z \equiv n(n-1) / 2(\bmod 2)$.

2. Proof of theorem. We first define a two-dimensional abstract algebraic complex $K[2$, p. 89]. The two-dimensional elements are the $(n)(n-1)$ triplets, $t$, of $\$ 1$. The one-dimensional elements are the $(3 / 2)(n)(n-1)$ ordered pairs $\left(t, P_{i} t\right)$, where, $\pi_{i} t=\left(m, m^{\prime}\right)$ with the restriction $m<m^{\prime}$. The zero-dimensional elements are the $n$ integers $m, 1 \leqq m \leqq n$.

We now define the incidence numbers. These are

$$
\begin{gathered}
{\left[t:\left(t, P_{i} t\right)\right]=1, \quad\left[P_{i} t:\left(t, P_{i} t\right)\right]=-1,} \\
{\left[\left(t, P_{i} t\right): m^{\prime}\right]=1, \quad\left[\left(t, P_{i} t\right): m\right]=-1 \text { if } \pi_{i} t=\left(m, m^{\prime}\right) .}
\end{gathered}
$$

All other incidence numbers are zero.

The diagram on the next page, indicating a partition of the torus into six triangles, is a geometric realization of the complex defined from the six triples $(1,2,3),(1,3,2),(2,1,3),(2,3,1),(3,1,2)$, $(3,2,1)$. (Compare to the example in $[1$, p. 222]).

Since the two-dimensional elements are not determined by their vertices, $K$ is certainly not a simplicial complex. However the derived complex $K^{\prime}[2$, p. 164] is simplicial.

Let us assume that $K^{\prime}$ is in fact represented as a polyhedron. We 


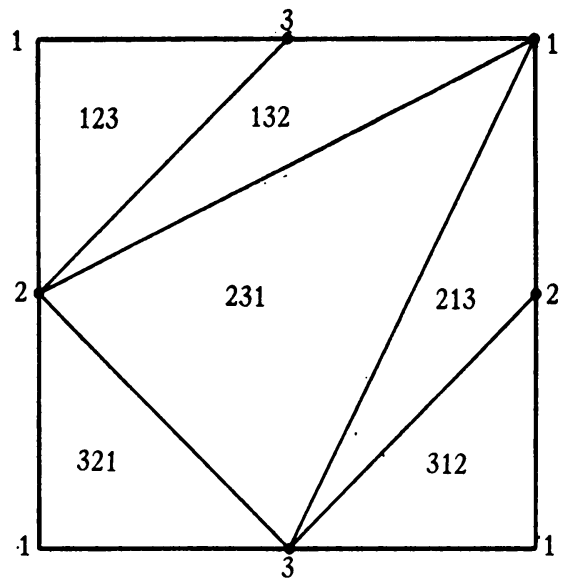

note that $K^{\prime}$ is connected, that each edge is incident to precisely two triangles, and that $K^{\prime}$ is orientable. Each vertex of $K^{\prime}$, not corresponding to the $n$ zero-dimensional elements of $K$, clearly has a disk neighborhood.

For $K^{\prime}$ to be a manifold, each of the vertices of $K^{\prime}$ corresponding to the $n$ zero-dimensional elements of $K$ must also have a disk neighborhood. Call these vertices in $K^{\prime}$ again $m, 1 \leqq m \leqq n$.

'This is equivalent to the assertion in $K^{\prime}$ that the edges and vertices of the closure of the star of $m$ (each $m, 1 \leqq m \leqq n$ ), and not incident to $m$, form the triangulation of a simple closed curve.

This assertion translates in $K$ into the condition that $\Phi(m)=1$. In general however $\Phi(m) \geqq 1$, so $K^{\prime}$ is not necessarily a manifold.

We obtain a manifold, $K^{\prime \prime}$, in the following manner, which we describe, for simplicity, in geometric terms. Remove from $K^{\prime}$ the $n$ vertices corresponding to vertices of $K$. Replace the vertex corresponding to the number $m$ by $\Phi(m)$ distinct symbols, replacing $m$ in each of the $\Phi(m)$ disks containing $m$, by one of these symbols. Then incorporate these symbols into a simplicial scheme by replacing the symbol $m$ in the incidence relations by the appropriate one of the $\Phi(m)$ symbols, depending on the disk in question. This process essentially consists of separating the $\Phi(m)$ disks at their common point $m$.

Let X stand for "Euler characteristic of." Since $K^{\prime \prime}$ is an orientable two-dimensional manifold, $\mathrm{X}\left(K^{\prime \prime}\right) \equiv 0(\bmod 2)$. But

$$
\begin{aligned}
\mathrm{X}\left(K^{\prime \prime}\right) & =Z-n+\mathrm{X}\left(K^{\prime}\right)=Z-n+\mathrm{X}(K) \\
& =Z-n+n-\frac{3}{2} n(n-1)+(n)(n-1) .
\end{aligned}
$$


Thus $Z \equiv(n)(n-1) / 2(\bmod 2)$ and Theorem II is proved.

3. Remarks. We first make some definitions. An $n \times n$ square array of elements $m, 1 \leqq m \leqq n$, is column-Latin if each column contains $n$ distinct elements $[3$, p. 335]. If, furthermore, the first row is the integers $m, 1 \leqq m \leqq n$, in their natural order the array is standard column-Latin.

If $A$ and $B$ are an orthogonal pair of standard column-Latin squares, we form a collection of triples satisfying (i), (ii) of Theorem I, by taking all $\left(b_{1}, b_{2}, b_{3}\right), 1 \leqq b_{i} \leqq n, i=1,2,3$ where $b_{2}$ and $b_{3}$ are in the same position in $A$ and $B$ and not in the first row and $b_{1}$ is their column index. This construction implies that there is a many-one correspondence between such pairs $A, B$ and diagonalized Latin squares. MacNeish applied this construction to orthogonal pairs of standard Latin squares in [1] to prove that there is no orthogonal pair of Latin squares of order $n \equiv 2(\bmod 4)$. The error in this proof is the statement "each of the $n$ vertices must occur in the same number of circuits," [2, p. 224]; for from this statement the conclusion is then drawn that $Z \equiv 0(\bmod n)$. Examples exist contradicting this latter assertion. It is interesting to note that the proof in [1] does not exploit the full Latin character of $A$ and $B$. The following is an example of a $6 \times 6$ diagonalized Latin square:

$\begin{array}{llllll}1 & 6 & 5 & 2 & 3 & 4 \\ 3 & 2 & 4 & 6 & 1 & 5 \\ 6 & 4 & 3 & 5 & 2 & 1 \\ 2 & 5 & 1 & 4 & 6 & 3 \\ 4 & 1 & 6 & 3 & 5 & 2 \\ 5 & 3 & 2 & 1 & 4 & 6\end{array}$

\section{REFERENCES}

1. Harris F. MacNeish, Euler squares, Ann. of Math. (2) vol. 23 (1921-1922) pp. 221-227.

2. Solomon Lefschetz, Algebraic topology, Amer. Math. Soc. Colloquium Publications, vol. 27.

3. Donald A. Norton, Groups of orthogonal row-Latin squares, Pacific Journal of Mathematics vol. 2 (1952) pp. 335-341.

University of California, Davis 